\title{
Time Preferences, Intertemporal Optimization, and the Permanent Income-Life Cycle Hypothesis
}

\author{
Hiroaki Hayakawa \\ Professor of Economics, School of Business and Economics, Universiti Brunei Darussalam, Brunei \\ Email:hayakawah2@,r4.dion.ne.jp
}

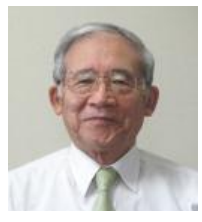

\begin{abstract}
This paper analyzes how the three lines of development, namely, the structure of dynamic recursive preferences, Pontryagin's maximum principle, and the permanent income-life cycle hypothesis, are interrelated in the context of consumer choice in continuous time. First, by introducing variational changes in consumption around two different points in time that are compensating to the lifetime utility or having canceling effects on asset accumulation, we define and compute the marginal rates of substitution and transformation and express the optimality condition in terms of the instantaneous time rates of change of these rates. This condition is then related to the Böhm-Bawerkian and Fisherian rates of time preference, which are defined, calculated, and shown to give an equivalent measure whether inclusive of the first order change of consumption or not. The Keynes-Ramsey rule of consumption is related to this optimality condition and is given a new interpretation. Second, we inquire into the preference basis of the permanent income-life cycle hypothesis and show explicitly that the proportionality of optimal consumption to wealth and permanent income follows from the homotheticity of recursive time preferences and a dynamic budget constraint, thereby confirming that the hypothesis, as originally conceived by Friedman and Modigliani-Brumberg, is a dynamic version of the static demand under homothetic preferences.
\end{abstract}

Keywords: Recursive preferences, Rate of time preference, Homotheticity, Proportionality, Permanent income, Maximum principle. JEL Classification: E2; E4; D9.

Citation | Hiroaki Hayakawa (2019). Time Preferences, Intertemporal Optimization, and the Permanent Income-Life Cycle Hypothesis. Growth, 6(1): 1-11.

\section{History:}

Received: 3 June 2019

Revised: 5 July 2019

Accepted: 8 August 2019

Published: 12 September 2019

Licensed: This work is licensed under a Creative Commons

Attribution 3.0 License $(\mathrm{cc})$ E

Publisher: Asian Online Journal Publishing Group
Funding: This study received no specific financial support

Competing Interests: The author declares that there are no conflicts of interests regarding the publication of this paper.

Transparency: The author confirms that the manuscript is an honest, Transparency: The author confirms that the manuscript is an honest,
accurate, and transparent account of the study was reported; that no vital accurate, and transparent account of the study was reported; that no vital
features of the study have been omitted; and that any discrepancies from the study as planned have been explained.

Ethical: This study follows all ethical practices during writing.

\section{Contents}

1. Introduction

2. Recursive Time Preferences

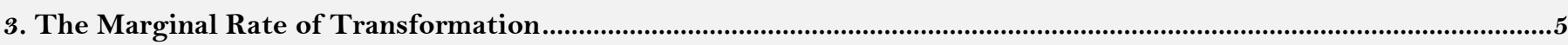

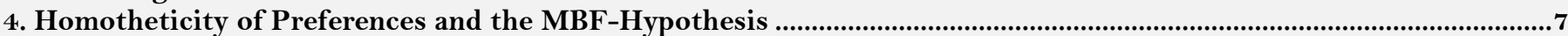

5. Conclusion 


\section{Contribution of this paper to the literature}

This study contributes to existing literature by examining how the three lines of development, namely, the structure of dynamic recursive preferences, Pontryagin's maximum principle, and the permanent income-life cycle hypothesis, are interrelated in the context of consumer choice in continuous time.

\section{Introduction}

Modigliani and Brumberg's life-cycle hypothesis (Modigliani and Brumbert, 1954) and Friedman's permanent income hypothesis (Friedman, 1957) (hereafter the MBF-hypothesis), were the forerunners of dynamic macroeconomic theory developed over the past several decades. The calculus of variation and the optimal control theory have contributed to the enrichment of the literature on this theory. Initially, the applications of these methods were restricted to the assumption that time preferences remain unchanged over time, but later, as the structure of such preferences was scrutinized in the pioneering works of Koopmans (1960); Koopmans et al. (1964) and Uzawa (1968) the preferences that are recursive in nature and which allow the rate of time preference to vary endogenously have enriched the analysis of intertemporal behavior (Blackorby et al., 1978; Epstein and Hynes, 1983; Epstein, 1987a;1987b; Obstfeld, 1990). At the same time, along with this development, the MBF-hypothesis has continued to inspire many generations of researchers (Sargent, 1987; Baranzini, 2005; Deaton, 2005). This is evident in a series of works published in recent years, that have reexamined the hypothesis from old and new perspectives, theoretically and empirically, and which contributed to better understanding of the validity of the hypothesis along with its limitations (e.g., (Hall, 1978; Flavin, 1981;1985; Campbell and Deaton, 1989; Runkle, 1991; Deaton, 1992; Attanasio, 1995; Hayashi, 1996; 1997; Seater, 1998; Serlenga, 2001; Dejuan and Seater, 2006; DeJuan et al., 2006; Omgba and Djiofack, 2010; Gomes, 2011; Tekin, 2011; Beznoska and Ochmann, 2012; Ni and Seol, 2014; Alimi, 2015; Kelikume et al., 2017; Noor et al., 2018)). But, there has been little work done that relates the hypothesis, as originally conceived, to the structure of intertemporal preferences under which an optimal consumption path is determined; this is particularly evident in the fact that the empirical testing of the hypothesis has been based on the constancy of the time preference. Hence, the question has been left unanswered as to the intertemporal preference basis of the hypothesis. This paper attempts to close part of this gap by elucidating this basis. This question is apart from Muth's point that Friedman's estimation of permanent income is optimal under a certain income-generating process or from stochastic implications of the hypothesis considered in Hall (1978) and subsequent works.

Specifically, this paper shows how the three lines of development, namely, the structure of recursive preferences of Koopmans-Uzawa-Epstein kind (Koopmans, 1960; Koopmans et al., 1964; Uzawa, 1968), the Hamiltonian method and Pontryagin's maximum principle as a tool to analyze dynamic behavior, and the MBFhypothesis are interrelated. In particular, (1) we define the Fisherian and the Böhm-Bawerkian rates of time preferences and show that they are equivalent, whether they are defined along locally constant paths or inclusive of the effect of the first order change of consumption. (2) We show how the instantaneous rate of change of the marginal rate of substitution is related to this rate of time preference. (2) Given a specific specification of the asset accumulation equation, the instantaneous rate of change of the marginal rate of transformation at any future point in time equals the real interest rate expected for that moment. (3) The optimal consumption requires that the instantaneous rate of change of the marginal rate of substitution be equal to that of the marginal rate of transformation at all points in time, from which the Keynes-Ramsey rule is obtained. (4) The homotheticity of intertemporal preferences implies that the rate of time preference is independent of the scale of consumption paths, which requires, for recursive time preferences, that the instantaneous discounting function be constant. (5) For homothetic preferences in general, the effect of the lifetime wealth on the optimal consumption path is proportional. This proportional wealth effect, in turn, necessitates that a utility functional representing such preferences be written with an iso-elastic instantaneous utility function. (6) With (4) and (5) combined, if preferences are homothetic, the utility functional is written with an iso-elastic utility function and a constant discounting function. (7) Finally, we show, by way of Pontryagin's maximum principle and under constant real interest rates, the MBFhypothesis follows from this particular form of a utility functional, that is, from the homotheticity of recursive time preferences. In particular, we show the condition under which permanent consumption is a fixed proportion of permanent income as was put forth by Friedman (1957).

Thus, the consumer behavior as captured by the MBF-hypothesis results from a particular specification of recursive preferences. It is this preference basis of the MBF-hypothesis that this paper attempts to show, among others, along together with the logic of intertemporal optimization made explicit in terms of the rate of time preference, the marginal utility of consumption, the marginal rate of substitution, and the marginal rate of transformation. Elucidation of this logic in continuous time is itself an important purpose of this paper.

\section{Recursive Time Preferences}

Consider the class $\Omega$ of consumption paths extending from time 0 to $\infty$, each of which is a continuously differentiable, bounded, and positive function of time.

Then, consider the class $\Gamma$ of all recursive time preferences that are defined on this set. For notation, a consumption path and its value at time time $t$ are denoted by $C$ and $c(t)$, and a path whose time $t$ consumption is equal to $c(T+t)$, is denoted by ${ }_{t} C$. This is a right-hand tail path obtained from $C$.

Each member of the class $\Gamma$ is represented by a utility functional of the form:

$$
U(C)=\int_{0}^{\infty} v(c) e^{-\int_{0}^{t} \delta(t) d \tau} d t
$$

Where $v(c)$ and $\delta(c)$ are assumed to be real-valued and twice continuously differentiable. The structure of recursive preferences and this utility functional representation of such preferences has been investigated by Koopmans (1960), Koopmans et al. (1964), Blackorby et al. (1978), Epstein and Hynes (1983), Epstein (1987a;1987b) 
and Obstfeld (1990). In the following analysis, $v(c)$ will be referred to as the instantaneous utility function, and $\delta(c)$ as the instantaneous discounting function. As related to the argument advanced by Becker and Mulligan (1997) on the endogenous effort to engage in activities that connect the current and future well-being, there is an important issue as to whether the marginal impatience is increasing or diminishing as a function of consumption, which relates to whether $\delta(c)$ is an increasing or a decreasing function of consumption. Here we allow both cases.

Implicit in the structure of $U(C)$ is the rate of time preference. Two different notions of this rate have been introduced and discussed in the literature. One is the Boehm-Bawerkian notion, due to Böhm-Bawerk (1988) which is based on the idea of preference for advancing the timing of future satisfaction and consists in quantifying how much the marginal utility of consumption at any future point in time will change if the timing of that consumption is advanced by a small amount of time.

The other is the Fisherian notion, due to Fisher (1930) which is based on the marginal rate of substitution between consumption at two different points in time and considers how much this rate will change as the timing of consumption of the second date is changed. We first show that these two notions give an identical measurement, regardless of whether it is measured along locally constant paths or inclusive of the effect of the first order change of consumption.

For either notion, we need to formalize the concept of the marginal utility of consumption (i.e., how much the lifetime utility, as measured at the point of decision making) changes with a small variational change of consumption around a particular future point in time). This can be done formally through use of the Volterra derivative (Volterra, 1959; Wan, 1970; Ryder and Heal, 1973; Hayakawa and Ishizawa, 1993;1997). In our derivation, we consider a uniform variational change after Uzawa (1990) and Imai et al. (1972).

This method simplifies the computational steps and elucidates the results reported in Epstein (1987a;1987b). The marginal utility of consumption derived this way gives the same results as the Volterra derivative as uniform variational changes are just a special form of variational changes in general (Hayakama and Ishizawa, 1997).

Given a consumption path $C$, we consider a small variational change in period $(T, T+\Delta t)$ such that consumption is higher uniformly by $\Delta c$ during that period, and construct a consumption path $C^{\prime}$ defined as:

$$
C^{\prime}=\left\{\begin{array}{c}
c(t), 0 \leq t<T \\
c(t)+\Delta c, T \leq t \leq T+\Delta t \\
c(t), T+\Delta t<t
\end{array}\right.
$$

The marginal utility of consumption at time $T$ as measured along path $C$ and evaluated at time 0 , denoted $M U_{T}(C)$, is defined and computed as:

$$
M U_{T}(C)=\lim _{\Delta c \rightarrow 0} \lim _{\Delta t \rightarrow 0} \frac{U\left(C^{\prime}\right)-U(C)}{\Delta c \Delta t}=e^{-\int_{0}^{T} \delta(t) d \tau}\left[v^{\prime}(c(T))-\delta^{\prime}(c(T)) U\left({ }_{T} C\right)\right]
$$

where $U\left({ }_{T} C\right)$ is the future lifetime utility as of time $T$, i.e.,

$$
U\left({ }_{T} C\right)=\int_{T}^{\infty} v(c(t)) e^{-\int_{T}^{t} \delta(s) d s} d t
$$

On the other hand, taking the derivative of $U\left({ }_{T} C\right)$ with respect to $T$ yields

$$
\frac{d}{d T} U\left({ }_{T} C\right)=-v(c(T))+\delta(c(T)) U_{T}(C) .
$$

If the timing of the above variational change, $T$, is delayed, the marginal utility will decline in general; hence, taking the negative of the rate of change of the marginal utility with respect to $T$, we obtain an equation with which to measure the preference for advancing the timing of future satisfaction. Thus, taking a logarithmic time derivative of $U\left({ }_{T} C\right)$ gives:

$$
\begin{gathered}
\hat{\xi}_{B}(T)=-\frac{d}{d T} \log U\left({ }_{T} C\right) \\
=\frac{\delta-\left(\frac{\delta^{\prime}}{v^{\prime}}\right) v}{1-e}-\left(\frac{\eta_{v-\eta_{\delta} e}}{1-e}\right) \frac{\dot{c}}{c} \\
\text { where } \\
e=\left(\frac{\delta^{\prime}(c)}{v^{\prime}(c)}\right) U\left({ }_{T} C\right) \\
\eta_{\delta}=\delta^{\prime \prime}(c) c / \delta^{\prime}(c)\left(\text { the elasticity of } \delta^{\prime}(c) \text { with respect to } c\right) \\
\eta_{v}=v^{\prime \prime}(c) c / v^{\prime}(c)\left(\text { the elasticity of } v^{\prime}(c) \text { with respect to } c\right)
\end{gathered}
$$

The dot over a variable denotes its time rate of change.

'Epstein (1987b) has shown that a generating function, which corresponds to Koopmans' aggregator function in discrete time Koopmans (1960) may be obtained from $(5)$ as

$$
\begin{aligned}
& \qquad \begin{array}{c}
f(c, \varphi)=v(c)-\delta(c) \varphi \\
\text { where }=U\left({ }_{T} C\right) \text {. From this function, the rate of time preference, } \xi_{B}(T) \text { or } \xi_{F}(T), \text { can be obtained as } \\
\xi_{B}(T)=\frac{f(c, \varphi) f_{c \varphi}}{f_{c}}-f_{\varphi} .
\end{array} \\
& \text { For Uzawa (1968) utility functional, the generating function is given by }
\end{aligned}
$$$$
\text { For Uzawa (1968) utility functional, the generating function is given by }
$$$$
f(c, \varphi)=v(c)-\delta(v(c)) \varphi \text {, }
$$

while, in the case of Epstein and Hynes (1983) functional, the same function reduces to

$$
f(c, \varphi)=-1-\delta(v(c)) \varphi \text {. }
$$


The rate of time preference, denoted $\hat{\xi}_{B}(T)$, is a measure of the preference for advancing the timing of future satisfaction. Since Equation 6 includes the effect of the first order change of consumption, we let the same rate be defined by netting this effect from Equation 6 i.e., by restricting $\hat{\xi}_{B}(T)$ to locally constant paths. Hence, setting $c^{*} / c=0$ in Equation 6 gives:

$$
\begin{gathered}
\xi_{B}(T)=\hat{\xi}_{B}(T)_{c^{\prime} / c=0} \\
=\left[\delta(c)-\left(\frac{\delta^{\prime}(c)}{v^{\prime}(c)}\right) v(c)\right] /(1-e), e=\left(\frac{\delta^{\prime}(c)}{v^{\prime}(c)}\right) U\left({ }_{T} C\right)
\end{gathered}
$$

These results are consistent with what is reported in Epstein (1987a;1987b). Thus, we have two measures of the rate of time preference, one along locally constant paths and the other inclusive of the first order change of consumption.

We note that in order for the rate of time preference computed in Equation 7 not to vanish, the condition: $\delta v^{\prime}-\delta^{\prime} v \neq 0$ (i.e., $\delta^{\prime} / \delta \neq v^{\prime} / v$ ) needs to be satisfied. In this connection, we make the following observation: The lifetime utility (1) converges in steady state to $v(c) / \delta(c)$ (the steady state welfare in terms of the lifetime utility). If this is differentiated with respect to $c$, it is seen that this welfare increases with consumption if and only if $\delta v^{\prime}-\delta^{\prime} v>0$.

This condition, therefore, assures that the rate of time preference does not vanish. But, if the rate is to be positive (on top of $\delta v^{\prime}-\delta^{\prime} v \neq 0$ ), we need a further restriction that $1-e>0$. The condition: $\delta v^{\prime}-\delta^{\prime} v \neq 0$ was stipulated by Uzawa (1968) who worked with an assumption that $\delta^{\prime}(c)>0$, which is the case of the marginal impatience increasing with consumption.

Moreover, in steady state, the rate of time preference as defined by (7) equals $\delta(c)$, which shows that if $\delta^{\prime}(c)>0$, the steady state rate of time preference increases with consumption. On the other hand, if $\delta^{\prime}(c)<0$, which is the case of the marginal impatience decreasing with consumption, i.e., the case of Becker and Mulligan (1997) we see that the conditions: $\delta v^{\prime}-\delta^{\prime} v>0$ and $1-e>0$ are both satisfied, so that the rate of time preference (7) is positive. In this case, the steady state welfare increases with consumption, and the steady state rate of time preference diminishes with consumption.

We now turn to the Fisherian notion of the rate of time preference. Again, let a consumption path $C$ be given. Because the notion is based on the marginal rate of substitution between two different points in time, we need to consider small variations of consumption during two short periods with duration $\Delta t$, say, at $T$ and $T^{\prime}:(T, T+\Delta t)$ and $\left(T^{\prime}, T^{\prime}+\Delta t\right)$, where consumption in the first period is reduced uniformly by $\Delta c$ and consumption in the second period increased likewise by $\Delta c^{\prime}$ so that the lifetime utility remains unchanged.

The marginal utilities at $T$ and $T^{\prime}$ are computed, as in Equation 3, as:

$$
\begin{aligned}
& M U_{T}(C)=e^{-\int_{0}^{T} \delta(t) d \tau}\left[v^{\prime}(c(T))-\delta^{\prime}(c(T)) U\left({ }_{T} C\right)\right] \\
& M U_{T^{\prime}}(C)=e^{-\int_{0}^{T} \delta(t) d \tau}\left[v^{\prime}\left(c\left(T^{\prime}\right)\right)-\delta^{\prime}\left(c\left(T^{\prime}\right)\right) U\left({ }_{T}, C\right)\right]
\end{aligned}
$$

On the other hand, the marginal rate of substitution between $T$ and $T^{\prime}$, denoted $R\left(T, T^{\prime}\right)$, is defined as:

$$
\begin{gathered}
R\left(T, T^{\prime}\right)=\left.\lim _{\Delta c \rightarrow 0} \lim _{\Delta t \rightarrow 0} \frac{\Delta c^{\prime}}{\Delta c}\right|_{U(C)=\text { constant }} \\
=e^{-\int_{T}^{T^{\prime}} \delta(c(\tau)) d \tau} \frac{v^{\prime}(c(T))-\delta^{\prime}(c(T)) U\left({ }_{T} C\right)}{v^{\prime}\left(c\left(T^{\prime}\right)\right)-\delta^{\prime}\left(c\left(T^{\prime}\right)\right) U\left(_{T^{\prime}} C\right)}
\end{gathered}
$$

The logarithm of Equation 10 equals:

$$
\begin{gathered}
\sigma\left(T, T^{\prime}\right) \equiv \log R\left(T, T^{\prime}\right) \\
=-\int_{T}^{T^{\prime}} \delta(c(\tau)) d \tau+\log \left\{v^{\prime}(c(T))-\delta^{\prime}(c(T)) U\left({ }_{T} C\right)\right\} \\
-\log \left\{v^{\prime}\left(c\left(T^{\prime}\right)\right)-\delta^{\prime}\left(c\left(T^{\prime}\right)\right) U\left({ }_{T^{\prime}} C\right)\right\}
\end{gathered}
$$

Hence, differentiating Equation 11 partially with respect to $T^{\prime}$ and evaluating the derivative at $T^{\prime}=T$ gives the instantaneous rate of change of the marginal rate of substitution of consumption at time $T$, denoted $\hat{\xi}_{F}(T)$.

$$
\hat{\xi}_{F}(T)=\left.\frac{\partial \sigma\left(T, T^{\prime}\right)}{\partial T^{\prime}}\right|_{T^{\prime}=T}=\frac{\delta(c)-\left(\frac{\delta^{\prime}(c)}{v^{\prime}(c)}\right) v(c)}{1-e}-\left(\frac{\eta_{v-} \eta_{\delta} e}{1-e}\right) \frac{\dot{c}}{c}, e=\left(\frac{\delta^{\prime}(c)}{v^{\prime}(c)}\right) U\left({ }_{T} C\right)
$$

Where $\left.(d / d T) U\left({ }_{T^{\prime}} C\right)\right|_{T^{\prime}=T}=-v(c(T))+\delta(c(T)) U\left({ }_{T^{\prime}} C\right)$ is substituted in. Note that since $\left.\frac{\partial \sigma\left(T, T^{\prime}\right)}{\partial T^{\prime}}\right|_{T^{\prime}=T}=$ $-\left.\frac{\partial \sigma\left(T, T^{\prime}\right)}{\partial T}\right|_{T=T^{\prime}}, \hat{\xi}_{F}(T)$ can be defined by the latter as well as by the former except the sign.

This is an expression identical, term by term, to Equation 6 above. Thus, it is seen that the negative of the instantaneous rate of change of the marginal utility of consumption at time $T$ (i.e., $=-\frac{d}{d T} \log U\left({ }_{T} C\right)$ ) equals the 
instantaneous rate of change of the marginal rate of substitution at time $T$ (i.e., either $\partial \sigma\left(T, T^{\prime}\right) /\left.\partial T^{\prime}\right|_{T^{\prime}=T}$ or $\left.-\partial \sigma\left(T, T^{\prime}\right) /\left.\partial T\right|_{T=T^{\prime}}\right)$.

The Fisherian rate of time preference at time $T$, denoted $\xi_{F}(T)$, is defined as the instantaneous rate of change of the marginal rate of substitution at time $T$ evaluated along locally constant paths. Hence, with $c^{*} / c=0$ imposed on $\hat{\xi}_{F}(T)$ at $T^{\prime}=T$, the Fisherian rate of time preference is obtained as:

$$
\xi_{F}(T)=\frac{\delta(c)-\left(\frac{\delta^{\prime}(c)}{v^{\prime}(c)}\right) v(c)}{1-e}, \text { where } e=\left(\frac{\delta^{\prime}(c)}{v^{\prime}(c)}\right) U\left({ }_{T} C\right)
$$

which is equivalent to the Böhm-Bawerkian rate of time preference given in Equation 7 above. Thus, we have established that the Böhm-Bawerkian and the Fisherian rates of time preference are equivalent to each other. ${ }^{2}$

The rate of time preference may be defined inclusive of the effect of the first order change of consumption. This alternative definition may cause some confusion via-a-vis the one that excludes this effect. Hence, in defining the rate, it should be made clear which measurement is chosen, along locally constant paths or inclusive of other effects. If the rate is defined inclusive of the effect of $\dot{c} / c$, then $\hat{\xi}_{B}(T)$ given in Equation 6 or $\hat{\xi}_{F}(T)$ given in Equation 12 should be used; Uzawa (1990) defines the rate of time preference in this fashion; see also Imai et al. (1972).

Under this definition, the rate can be treated as equivalent to the negative of the instantaneous rate of change of the marginal utility of consumption as well as to the instantaneous rate of change of the marginal rate of substitution of consumption. On the other hand, if the restriction is imposed that the rate be measured along locally constant paths, $\xi_{B}(T)$ given in Equation 7 or $\xi_{F}(T)$ given in Equation 13 is the relevant quantity to be used; this is the way Epstein and Hynes (1983) and Epstein (1987a;1987b) define the rate of time preference. Under the restriction, however, the equivalence between the rate of time preference and the instantaneous rate of change of the marginal utility (with minus sign) or the marginal rate of substitution is lost. Since the underlying Equations 6 and 12 are identical, no matter how the rate of time preference may be defined (whether along locally constant paths or inclusive of the effect of the first order change of consumption), there should be no difference between the Böhm-Bawerkian and Fisherian rates.

In this paper, these rates are defined along locally constant paths, but this is a matter of choice. Evidently, the difference between $\hat{\xi}_{B}(T)$ and $\xi_{F}(T)$ or between $\hat{\xi}_{F}(T)$ and $\xi_{F}(T)$ disappear in steady state.

\section{The Marginal Rate of Transformation}

We now turn to the marginal rate of transformation. Determination of this rate requires that the asset accumulation process be specified. First, we need to define what this rate is.

Suppose that some asset accumulation process $A$ (i.e., a path of the stock of real assets) is given, and that this path is supported by a consumption path $C$. Take two periods of duration $\Delta t$, i.e., $(T, T+\Delta t)$ and $\left(T^{\prime}, T^{\prime}+\Delta t\right)$, and let consumption be reduced in the first period by $\Delta c$ and increased in the second period by $\Delta c^{\prime}$ so as to have the same amount of assets accumulated by the end of the second period as without such changes. Since consumption is reduced in the first period, the stock of assets grows faster relative to path $A$ during the period, and its growth rate starts to fall at time $T+\Delta t$ as consumption returns to the same level as its original path. Finally, with the increased consumption, the amount of real assets accumulated by time $T^{\prime}+\Delta t$ returns to the same level as along path $A$.

The stock of real assets, $a_{t}$, remains above the original path between time $T$ and time $T^{\prime}+\Delta t$ at which it returns to the same level as before the changes.

Such changes in consumption during the two periods make it possible to define the rate of transformation between time $T$ and $T^{\prime}$, denoted $N\left(T, T^{\prime}\right)$, as follows:

$$
N\left(T, T^{\prime}\right)=\lim _{\Delta c \rightarrow 0} \lim _{\Delta t \rightarrow 0} \frac{\Delta c^{\prime}}{\Delta c}
$$

The rate of change of $N\left(T, T^{\prime}\right)$ with respect to $T^{\prime}$ evaluated at $T^{\prime}=T$ gives the instantaneous rate of change of the marginal rate of transformation at time $T$. We denote this rate of change by $s(T)$ :

$$
s(T)=\left.\left(\frac{\partial}{\partial T^{\prime}}\right) \log N\left(T, T^{\prime}\right)\right|_{T^{\prime}=T}
$$

We analyze next how this marginal rate of transformation and its instantaneous rate of change are determined in the context of a specific process of asset accumulation. Consider the following process employed by Imai et al. (1972); Takayama (1985) and Uzawa (1990). Subsequently, we will use this process to discuss the relationship between the homotheticity of recursive preferences and the MBF-hypothesis.

Suppose that a household earns income from two sources - labor and a single interest earning asset. Let $W_{t}$ and $i_{t}$ stand for the nominal wages and the nominal interest rate, respectively. With these sources, the household's nominal income at time $t$, denoted $Y_{t}$, is given by

$$
Y_{t}=W_{t}+i_{t} A_{t}
$$

where $A_{t}$ is the stock of the asset at time $t$ in nominal terms. This income is then allocated between consumption $C_{t}$ and asset accumulation $\dot{A}_{t}$, so that: 


$$
\dot{A}_{t}=Y_{t}-C_{t}
$$

Equation 17 can be transformed into an analogous expression in real terms. Let the corresponding quantities in real terms be denoted by small case letters: $y_{t}=Y_{t} / P_{t}, w_{t}=W_{t} / P_{t}$, and $c_{t}=C_{t} / P_{t}$ where $P_{t}$ is the price level. Also, let the inflation rate be $\pi_{t}=\dot{P}_{t} / P_{t}$. Using these notations, we may rewrite Equation 17 as:

$$
\begin{gathered}
\quad \dot{a}_{t}=y_{t}-c_{t} \\
=w_{t}+r_{t} a_{t}-c_{t} \text { where } r_{t}=i_{t}-\pi_{t} \text { (the real interest rate) }
\end{gathered}
$$

Then the marginal rate of transformation can be obtained as follows (Imai et al., 1972): First, rewrite Equation 18 as:

$$
\dot{a}_{t}-r_{t} a_{t}=w_{t}-c_{t}
$$

Then, multiply both sides of Equation 19 by $e^{-\int_{0}^{t} r_{\tau} d \tau}$ to get:

$$
\left(\frac{d}{d t}\right)\left(a_{t} e^{-\int_{0}^{t} r_{\tau} d \tau}\right)=\left(w_{t}-c_{t}\right) e^{-\int_{0}^{t} r_{\tau} d \tau}
$$

where $r_{\tau}$ is the real interest rate at time $\tau$. Integrating Equation 20 from 0 to $\infty$ and imposing the terminal condition: $\lim _{t \rightarrow \infty} a_{t} e^{-\int_{0}^{t} r_{\tau} d \tau}=0$ yields:

$$
\int_{0}^{\infty} c_{t} e^{-\int_{0}^{t} r_{\tau} d \tau} d t=a_{0}+\int_{0}^{\infty} w_{t} e^{-\int_{0}^{t} r_{\tau} d \tau} d t
$$

where $a_{0}=A_{0} / P_{0} ; a_{0}$ is the initial stock of real assets.

In determining the instantaneous rate of change of the marginal rate of transformation, we consider again two periods, $[T, T+\Delta t]$ and $\left[T^{\prime}, T^{\prime}+\Delta t\right]$, in which consumption is reduced by $\Delta c$ in the first period and increased by $\Delta c^{\prime}$ in the second period so as to leave the stock of real assets accumulated by time $T^{\prime}+\Delta t$ unchanged. With such changes in consumption, 21 can be written as:

$$
\begin{aligned}
\int_{0}^{\infty} c_{t} e^{-\int_{0}^{t} r_{\tau} d \tau} d t & -\int_{T}^{T+\Delta t}(\Delta c) e^{-\int_{0}^{t} r_{\tau} d \tau} d t+\int_{T^{\prime}}^{T^{\prime}+\Delta t}\left(\Delta c^{\prime}\right) e^{-\int_{0}^{t} r_{\tau} d \tau} d t \\
& =a_{0}+\int_{0}^{\infty} w_{t} e^{-\int_{0}^{t} r_{\tau} d \tau} d t
\end{aligned}
$$

which gives

$$
(\Delta c) \int_{T}^{T+\Delta t} e^{-\int_{0}^{t} r_{\tau} d \tau} d t=\left(\Delta c^{\prime}\right) \int_{T^{\prime}}^{T^{\prime}+\Delta t} e^{-\int_{0}^{t} r_{\tau} d \tau} d t
$$

Hence, the marginal rate of transformation between $T$ and $T^{\prime}$ is computed as:

$N\left(T, T^{\prime}\right)=\lim _{\Delta c \rightarrow 0} \lim _{\Delta t \rightarrow 0}\left(\frac{\Delta c^{\prime}}{\Delta c}\right)=\frac{\lim _{\Delta t \rightarrow 0}\left(\frac{1}{\Delta t}\right) \int_{T}^{T+\Delta t} e^{-\int_{0}^{t} r_{\tau} d \tau} d t}{\lim _{\Delta t \rightarrow 0}\left(\frac{1}{\Delta t}\right) \int_{T^{\prime}}^{T^{\prime}+\Delta t} e^{-\int_{0}^{t} r_{\tau} d \tau} d t}$

Thus, taking the logarithm of Equation 24 and partially differentiating it with respect to $T^{\prime}$ and then evaluating this derivative at $T^{\prime}=T$, we obtain that the instantaneous rate of change of the marginal rate of transformation at time $T$, denoted $\varepsilon(T)$, equals the value of the expected real interest rate for time $T$, i.e., $r_{T}$.

$$
\varepsilon(T)=\left.\left(\frac{\partial}{\partial T^{\prime}}\right) \log N\left(T, T^{\prime}\right)\right|_{T^{\prime}=T}=r_{T}
$$

We note that the same result can also be obtained by differentiating Equation 24 partially with respect to time $T^{\prime}$ and taking the obtained derivative to the limit as $T^{\prime} \rightarrow T$.

In achieving the intertemporal optimality, an agent must equate the instantaneous rate of change of the marginal rate of substitution, $\hat{\xi}_{B}(T)$, with the instantaneous rate of change of the marginal rate of transformation, $\varepsilon(T)$, at every point in time. That is, an agent should observe:

$$
\hat{\xi}_{B}(t)=\varepsilon(t) \text { at all } t
$$

Note that this optimality condition is stated in terms of the rates of change of the marginal rate of transformation and the marginal rate of substitution, not in terms of the levels of these marginal rates. This distinction has not been made carefully in the literature as the rule is usually stated in terms of equating the marginal rate of substitution with the marginal rate of transformation.

Moreover, if the rate of time preference is defined inclusive of $c^{*} / c$, condition (26) calls for equating this rate of time preference with the instantaneous rate of change of the marginal rate of transformation; otherwise, the left side must be decomposed into two parts, that is, the rate of time preference defined along locally constant paths and the effect of the rate of change of consumption, as in Equation 27 below. 


$$
r_{t}=\xi_{B}(t)-d\left(c^{*} / c\right) \text { where } d=\left(\eta_{v}-\eta_{\delta}\right) /(1-e),
$$

where it is assumed that the expected real interest rate at time $t$ (for time $t$ ) equals the real interest rate prevailing at time $t$ - the weak consistency axiom of Turnovsky and Burmeister (1977).

The decomposition of $\hat{\xi}_{B}(t)$ into $\xi_{B}(t)$ and $d\left(c^{*} / c\right)$ allows the dynamics of $c^{*} / c$ to be described as

$$
c^{\prime} / c=\vartheta\left[r_{t}-\xi_{B}(t)\right] \text { where } \vartheta=1 / d,
$$

which shows that, to the extent that $d$ is positive, the rate of consumption increases (decreases) whenever $r_{t}$ is greater (less) than the rate of time preference defined by $\xi_{B}(t)$; see Epstein and Hynes (1983) for this interpretation.

If $\delta(c)$ in Equation 1 is constant as in Ramsey (1928); Koopmans (1960) and Cass (1965), say, at $\beta$, then the rate of time preference $\xi_{B}(t)$ equals $\beta$, and $d$ becomes the elasticity of the instantaneous marginal utility $v^{\prime}(c)$. Hence, Equation 27 further reduces to:

$$
r_{t}=\beta-\eta_{v}\left(c^{*} / c\right)
$$

This is the well-known Keynes-Ramsey rule of intertemporal optimization. It shows that this rule is the same as the condition that the rate of time preference inclusive of the first order of change of consumption (given on the right side of Equation 29) is equal, at every point of time, to the instantaneous rate of change of the marginal rate of transformation, which is equal to the (expected) real interest rate (given on the left side Equation 29).

\section{Homotheticity of Preferences and the MBF-Hypothesis}

We now address the question of whether consumer behavior captured by the MBF-hypothesis follows from the homotheticity of recursive preferences. To answer the question, we first show that if preferences are homothetic, the rate of time preference defined along locally constant paths must be constant. We start with the definition of homotheticity:

Definition: (Homotheticity)

Preferences are said to be homothetic if for any two arbitrary consumption paths, $C^{1}$ and $C^{2}, C^{1}$ is preferred to $C^{2}$ if and only if $\lambda C^{1}$ is preferred to $\lambda C^{2}$ for any positive number $\lambda$.

Consider a consumption path $C$ and two periods, $[T, T+\Delta t]$ and $\left[T^{\prime}, T^{\prime}+\Delta t\right]$, with two compensating changes in consumption as before. That is, in addition to path $C$, we consider another path $C^{\prime}$ defined by:

$$
C^{\prime}=\left\{\begin{array}{c}
c(t), 0 \leq t \leq T \\
c(t)-\Delta c, T \leq t \leq T+\Delta t \\
c(t), T+\Delta t \leq t \leq T^{\prime} \\
c(t)+\Delta c^{\prime}, T^{\prime} \leq t \leq T^{\prime}+\Delta t \\
c(t), t>T^{\prime}+\Delta t
\end{array}\right.
$$

By construction, it holds that $U(C)=U\left(C^{\prime}\right)$. Multiply paths $C$ and $C^{\prime}$ by $\lambda>0$ to get new paths $\lambda C$ and $\lambda C^{\prime}$. If preferences are homothetic, $U(C)=U\left(C^{\prime}\right)$ implies $U(\lambda C)=U\left(\lambda C^{\prime}\right)$.

But, this means that along path $\lambda C$ the changes of consumption by $-\lambda \Delta c$ and $\lambda \Delta c^{\prime}$ in the two periods are also compensating changes. Hence, computing the marginal rate of substitution of consumption between $T$ and $T^{\prime}$ along path $\lambda C^{\prime}$ gives:

$$
\begin{gathered}
R\left(T, T^{\prime}\right)=\lim _{\Delta c \rightarrow 0} \lim _{\Delta t \rightarrow 0}\left(\frac{\lambda \Delta c^{\prime}}{\lambda \Delta c}\right)=\lim _{\Delta c \rightarrow 0} \lim _{\Delta t \rightarrow 0}\left(\frac{\Delta c^{\prime}}{\Delta c}\right) \\
= \\
\left(e^{-\int_{T}^{T^{\prime}} u(c(\tau)) d \tau}\right)\left\{\frac{v^{\prime}(c(T))-\delta^{\prime}(c(T)) U\left({ }_{T} C\right)}{v^{\prime}\left(c\left(T^{\prime}\right)\right)-\delta^{\prime}\left(c\left(T^{\prime}\right)\right) U\left(_{T^{\prime}} C\right)}\right\}
\end{gathered}
$$

Suppose that $C^{*}$ is the optimal path in $S$, so that $C^{*}$ is at least as good as any other path in $S$. Since $\lambda C^{*}$ is in $S(\lambda)$, we compare $\lambda C^{*}$ with any other path $C$ in $S(\lambda)$, which can be written as $\lambda C^{\prime}$ for some path $C^{\prime}$ in $S$. If preferences are homothetic, then $\lambda C^{*}$ must be at least as good as $\lambda C^{\prime}$.

Thus, $\lambda C^{*}$ is at least as good as any other path in $S(\lambda)$; i.e., $\lambda C^{*}$ is the optimal path in $S(\lambda)$. This shows that the effect of the lifetime wealth on the optimal consumption path is proportional; see also Pollak (1970).

However, this proportional wealth effect implies that the instantaneous utility function $v(c)$ is iso-elastic, i.e., that $v(c)$ is in the form of logarithm or some power function, or in the form of their linear transformation; see Yaari (1964) and Blinder (1974). Thus, altogether, the homotheticity of recursive preferences implies that the utility functional $U(C)$ takes the following form:

$$
\begin{aligned}
& U(C)=\int_{0}^{\infty} v(c(t)) e^{\beta t} d t \\
& v(c)=\left\{\begin{array}{c}
\alpha c^{k}+\gamma \text { if } 0<k<1 \\
\alpha \log c+\gamma \text { if } k=0
\end{array}\right.
\end{aligned}
$$

We now look into how this form of preferences is related to the MBF-hypothesis. We answer this question in the framework of optimal control theory using a particular asset accumulation process discussed above. 
Soon after the MBF-hypothesis was introduced, Farrell (1959) summarized it in an abstract form. This abstraction contains a good insight as we show.

The MBF-hypothesis (Farrell's version) (Farrell, 1959).

Normal income hypothesis:

In any given period, an individual agent's current income $y$ affects his consumption $c$ only through its effect

on his normal income $Y$. We may write this relationship as:

$c=f(Y)$, where $f(\cdot)$ is independent of current income $y$ and assets.

The proportionality hypothesis:

For any individual agent, the relationship between his consumption and his normal income is one of

proportionality.

We now show that this normal income is interest return on lifetime wealth and that the optimal current consumption is proportional to this income with the proportionality factor being a negative function of real interest rate (Imai et al., 1972; Takayama, 1985; Uzawa, 1990). What is of great interest here is that the MBF-hypothesis can be derived from the homotheticity of recursive preferences.

Given paths $\left\{W_{t}\right\}_{0}^{\infty},\left\{P_{t}\right\}_{0}^{\infty},\left\{i_{t}\right\}_{0}^{\infty}$ of the nominal wage rate, the price level, and the nominal interest rate, an agent's optimization problem is stated as:

$$
\text { Maximize } U(C)=\int_{0}^{\infty} v(c(t)) e^{-\beta t} d t
$$

where

$$
v(c)=\left\{\begin{array}{c}
\alpha c^{k}+\gamma \text { if } 0<k<1 \\
\alpha \log c+\gamma \text { if } k=0
\end{array}\right.
$$

subject to:

$\dot{a}_{t}=w_{t}+r_{t} a_{t}-c_{t}$

$c_{t} \geq 0$

We write the discounted value Hamiltonian as:

$$
H\left(c_{t}, a_{t}, \lambda_{t}\right)=\left[v\left(c_{t}\right)+\lambda_{t}\left(w_{t}+r_{t} a_{t}-c_{t}\right)\right] e^{-\beta t}
$$

Pontryagin's maximum principle gives:

$$
v^{\prime}(c)=\lambda_{t}
$$

$$
\dot{\lambda}_{t} / \lambda_{t}=\beta-r_{t}
$$

The transversality condition is stated as:

$$
\lim _{t \rightarrow \infty} \lambda_{t} a_{t} e^{-\beta t}=0
$$

Taking the logarithmic time derivative of Equation 37 gives

$$
\dot{\lambda}_{t} / \lambda_{t}=-\eta_{v}(\dot{c} / c)
$$

where $\eta_{v}=v^{\prime \prime}(c) c / v^{\prime}(c)$ is the elasticity of the marginal instantaneous utility $v^{\prime}(c)$, which is either $1-k$ or 1. Combining this with Equation 38, we obtain the Keynes-Ramsey rule:

$$
\beta+\eta_{v}(\dot{c} / c)=r_{t}
$$

The left hand side is the instantaneous rate of change of the marginal rate of substitution, and the right hand side is the instantaneous rate of change of the marginal rate of transformation.

If the real interest rate $r_{t}$ can be assumed to remain constant at $r$ for all $t$, Equation 41 implies that $c(t)$ changes over time at some constant rate $\theta$ where $\theta=(r-\beta) / \eta_{v}$ :

$$
c(t)=c_{0} e^{\theta t}
$$

where $c_{0}$ is the level of consumption at time 0 .

Assuming that $r>0$ to ensure that $\int_{0}^{\infty} c_{t} e^{-r t} d t$ converges, we combine Equations 42 and 21 to get

$$
c_{0}=(r-\theta) a_{0}^{*}
$$

where $a_{0}^{*}$ is the lifetime wealth existing at time 0 defined by

$$
a_{0}^{*}=a_{0}+\int_{0}^{\infty} w_{t} e^{-r t} d t
$$


Defining permanent income at time 0 as $y_{0}^{*}=r a_{0}^{*}$ and also the propensity to save as

$$
s\left(r ; \beta, \eta_{v}\right)=\theta / r=(1-\beta / r) / \eta_{v}
$$

where $\beta$ and $\eta_{v}$ are viewed as parameters, we may rewrite Equation 43 as

$$
c_{0}=\varsigma\left(r ; \beta, \eta_{v}\right) y_{0}^{*}
$$

where $\varsigma\left(r ; \beta, \eta_{v}\right)=1-s\left(r ; \beta, \eta_{v}\right) ; \partial \varsigma / \partial r<0, \partial \varsigma / \partial \beta>0, \partial \varsigma / \partial \eta_{v}>0$.

The necessary and sufficient condition for the propensity to save, or, identically, for the propensity to consume, to lie between 0 and 1 (i.e., $0<s\left(r ; \beta, \eta_{v}\right)<1$ or $0<\varsigma\left(r ; \beta, \eta_{v}\right)<1$ is given by

$$
r>\beta>\left(1-\eta_{v}\right) r
$$

under which consumption is increasing over time (i.e., $\theta=(r-\beta) / \eta_{v}>0$, which is consistent with Takayama (1985). ${ }^{3}$ We observe that if the real interest rate happens to equal the fixed rate of time preference, consumption takes a constant value across time; that is, consumption equals the permanent income $y_{0}^{*}=r a_{0}^{*}$, hence is proportional to this income with the proportionality factor of 1 .

But, as Friedman argued, consumption is a certain fixed fraction of permanent income (around 90\%), and his argument is consistent with the case that $r$ is greater than $\beta$, in which case, consumption is increasing. This point has not been made explicit in the literature.

Equation 46 shows that the optimal current level of consumption is proportional to permanent income with the proportionality factor $\varsigma\left(r ; \beta, \eta_{v}\right)$ and that this factor is a decreasing function of the real interest rate $r$ and an increasing function of both the discount rate $\beta$ and the elasticity of the marginal instantaneous utility $\left(\eta_{v}\right)$. Under the homotheticity of recursive preferences, the instantaneous discounting function is constant, and $\eta_{v}$ is also constant; hence, under these properties, the proportionality factor $\varsigma\left(r ; \beta, \eta_{v}\right)$ is constant, which is consistent with Friedman's assumption that permanent consumption is a constant proportion of permanent income. The same equation also shows that the elasticity of this optimal consumption with respect to either total lifetime wealth $a_{0}^{*}$ or permanent income $y_{0}^{*}$ is unitary.

Thus, the MBF-hypothesis can be obtained as a result of the homotheticity of recursive preferences. This preference basis of the MBF-hypothesis is in agreement with the static demand proposition that under static homothetic preferences, the quantity demanded of any good demanded is a fixed proportion of income or wealth where this proportion is determined by the prices of goods.

\section{Conclusion}

This paper examined the relationship between the structure of recursive preferences, Pontryagin's maximum principle, and the permanent income-life cycle hypothesis. We defined and computed the two crucial rates for intertemporal optimization: the marginal rate of substitution and the marginal rate of transformation, by considering compensating or offsetting variations with respect to the lifetime utility or the asset accumulation process, showing that the optimal consumer choice is characterized in terms of the instantaneous rates of change of these rates.

We also showed how the Böhm-Bawerkian and the Fisherian rates of time preference (which differ in conception) are computed, demonstrating that they give rise to an identical measurement as well as demonstrating how this rate of time preference is related to the optimality condition and the Keynes-Ramsey rule. We then examined how the permanent income-life cycle hypothesis is related to the homotheticity of recursive preferences under a particular asset accumulation equation, showing that the proportionality of consumption to wealth and permanent income, which is the core feature of the hypothesis, results from the preference homotheticity combined with an asset accumulation equation (a dynamic budget constraint).

Thus, the MBF-hypothesis is a dynamic version of the static consumer choice under homothetic preferences, which says that the quantity demanded of a good is proportional to income or wealth of the budget constraint while the proportionality factor is determined by the prices of goods.

Our demonstration makes the point that the homotheticity of recursive time preferences restricts the forms of the instantaneous discounting and utility functions that constitute such preferences, and that these restricted forms, together with a dynamic budget constraint, bring about the proportionality of optimal consumption to wealth or permanent income as conceived in the MBF-hypothesis.

We also made it clear that Freedman's proposition that permanent consumption is a fraction of permanent income holds when the market interest rate is above the rate of time preference which remains fixed under homothetic preferences, i.e., when the optimal consumption is rising.

\footnotetext{
3 Two special cases were considered in Imai et al (1972) and Uzawa (1990). The first one deals with constant real wage rates. With this assumption, lifetime wealth $a_{0}^{*}$ and permanent income $y_{0}^{*}$ equal, respectively, $a_{0}+w_{0} / r$ and $r\left(a_{0}+w_{0} / r\right)=r a_{0}+w_{0}$, which implies that permanent income coincides with current income. The second one allows real wage rates to grow at some constant rate $\alpha$, so that $w_{t}=w_{0} e^{\alpha t}$. In such a case, lifetime wealth $a_{0}^{*}$ equals $a_{0}+w_{0}(r-\alpha)$, and permanent income $y_{0}^{*}$ is given by $r a_{0}+r w_{0}(r-\alpha)$, so that the discrepancy between permanent and current income amounts to $y_{0}^{*}-$
} $y_{0}=\alpha w_{0} /(r-\alpha)$. 


\section{References}

Alimi, R., 2015. Estimating consumption function under permanent income hypothesis: A comparison between Nigeria and South Africa. International Journal of academic Research in Business and Social Sciences(5): 11.Available at: https://doi.org/10.6007/ijarbss/v5i $11 / 1917$.

Attanasio, O.P., 1995. The intertemporal allocation of consumption: Theory and evidence. Carnegie-Rochester Conference Series on Public Policy, 42 (1), 39-89.

Baranzini, M., 2005. Modigliani's life-cycle theory of savings fifty years later. BNL Quarterly Review, 58(233-234): 109-172.

Becker, G.S. and C.B. Mulligan, 1997. The endogenous determination of time preference. The Quarterly Journal of Economics, 112(3): 729758.

Beznoska, M. and R. Ochmann, 2012. Liquidity constraints and the permanent income hypothesis. Working Paper, German Institute for Economic Research. Available from https://pdfs.semanticscholar.org/bd50/05fb46d882933862baf59ddo89fb53c455b8.pdf.

Blackorby, C., D. Primont and R. Russell, 1978. Duality, separability, and functional structure: Theory and economic applications. New York: Elsevier.

Blinder, A.S., 1974. Toward an economic theory of income distribution. Cambridge, MA: MIT Press.

Böhm-Bawerk, E.V., 1988. Positive Theorie des Kapitals, translated with a preface and analysis by W. Smart (1930). New York: G.E. Stechert \& Co.

Campbell, J. and A. Deaton, 1989. Why is consumption so smooth? The Review of Economic Studies, 56(3): 357-373.Available at: https://doi.org/10.2307/2297552.

Cass, D., 1965. Optimum growth in an aggregative model of capital accumulation. The Review of Economic Studies, 32(3): 233-240.Available at: https://doi.org/10.2307/2295827.

Deaton, A., 1992. Understanding consumption. Oxford: Oxford University Press.

Deaton, A., 2005. Franco Modigliani and the life cycle theory of consumption. BNL Quarterly Review, 58(233-234): 91-107.

Dejuan, J. and J. Seater, 2006. A simple test of friedman's permanent income hypothesis. Economica, 73(289): 27-46.Available at: https://doi.org/10.1111/j.1468-0335.2006.00446.x.

DeJuan, J.P., J.J. Seater and T.S. Wirjanto, 2006. Testing the permanent-income hypothesis: New evidence from West-German states (Länder). Empirical Economics, 31(3): 613-629.Available at: https://doi.org/10.1007/s00181-005-0035-4.

Epstein, L.G., 1987a. A simple dynamic general equilibrium model. Journal of Economic Theory, 41(1): 68-95.

Epstein, L.G., 1987b. The global stability of efficient intertemporal allocations. Econometrica, 55(2): 329-355.

Epstein, L.G. and J.A. Hynes, 1983. The rate of time preference and dynamic economic analysis. Journal of Political Economy, $91(4): 611-$ 635 .

Farrell, M.J., 1959. The new theories of the consumption function. The Economic Journal, 69(276): 678-696.Available at: https://doi.org/10.2307/2227665.

Fisher, I., 1930. The theory of interest. New York: Macmillan.

Flavin, M.A., 1981. The adjustment of consumption to changing expectations about future income. Journal of Political Economy, 89(5): 9741009.Available at: https://doi.org/10.1086/261016.

Flavin, M., 1985. Excess sensitivity of consumption to current income: Liquidity constraints or myopia? Canadian Journal of Economics, 18(1): 117-136.Available at: https://doi.org/10.2307/135117.

Friedman, M., 1957. A theory of the consumption function. Princeton, NJ: Princeton University Press.

Gomes, F.A.R., 2011. Testing the permanent income hypothesis using unit root quantile autoregression tests. Applied Economics Letters, 18(18): 1755-1758.Available at: https://doi.org/10.1080/13504851.2011.562156.

Hall, R.E., 1978. Stochastic implications of the life cycle-permanent income hypothesis: Theory and evidence. Journal of Political Economy, 86(6): 971-987.Available at: https://doi.org/10.1086/260724.

Hayakama, H. and S. Ishizawa, 1997. The fundamentals of intertemporal optimization in the continuous time modelling of consumer behaviour. The Japanese Economic Review, 48(1): 101-112.Available at: https://doi.org/10.1111/1468-5876.00039.

Hayakawa, H. and S. Ishizawa, 1993. The fundamental principle of intertemporal optimization: Consumer behavior under recursive preferences. Economics Letters, 41(3): 273-280.Available at: https://doi.org/10.1016/0165-1765(93)90153-4.

Hayashi, F., 1996. Analysis of household saving: Past, present, and furture. The Japanese Economic Review, 47(1): 21-33.Available at: https://doi.org/10.1111/j.1468-5876.1996.tbo0033.x.

Hayashi, F., 1997. Understanding saving: Evidence from the U.S. and Japan. Cambridge, MA: MIT Press.

Imai, K., H. Uzawa, R. Komiya, Negishi and Y. Murakami, 1972. Price theory III. Tokyo: Iwanami (in Japanese).

Kelikume, I., F. Alabi and F. Anetor, 2017. Nigeria consumption function-an empirical test of the permanent income hypothesis. Journal of Global Economics, Management and Business Research, 9(1): 17-24.

Koopmans, T.C., 1960. Stationary ordinal utility and impatience. Econometrica: Journal of the Econometric Society, 28(2): 287-309.Available at: https://doi.org/10.2307/1907722

Koopmans, T.C., P.A. Diamond and R.E. Williamson, 1964. Stationary utility and time perspective. Econometrica, 32(1-2): 82-100.Available at: https://doi.org/10.2307/1913736.

Modigliani, F. and R.H. Brumbert, 1954. Utility analysis and the consumption function: An interpretation of cross-section data. In K. K. Kurihara (Ed.), Post-Keynesian Economics. New Brunswick, NJ: Rutgers University Press. pp: 388-436.

Ni, S. and Y. Seol, 2014. New evidence on excess sensitivity of household consumption. Journal of Monetary Economics, 63: 80-94.Available at: https://doi.org/10.1016/j.jmoneco.2014.01.004.

Noor, H., D. Lunfang, I. Khan and K. Khan, 2018. Testing of the empirical validity of permanent income hypothesis and absolute income hypothesis for China. Empirical Economics Letters, 17(4): 453-460.

Obstfeld, M., 1990. Intertemporal dependence, impatience, and dynamics. Journal of Monetary Economics, 26(1): 45-75.

Omgba, L.D. and C. Djiofack, 2010. Does permanent income hypothesis a solution? An empirical assessment via a dynamic general equilibrium model in developing country. Available from https://pdfs.semanticscholar.org/c86b/Odb4904121b5ca034167881062e6a81dd8e2.pdf.

Pollak, R.A., 1970. Homogeneous von neumann-morgenstern utility functions. International Economic Review, 11(1): 117-130.Available at: https://doi.org/10.2307/2525341.

Ramsey, F.P., 1928. A mathematical theory of saving. Economic Journal, 38(152): 543-559.Available at: https://doi.org/10.2307/2224098.

Runkle, D.E., 1991. Liquidity constraints and the permanent-income hypothesis: Evidence from panel data. Journal of Monetary Economics, 27(1): 73-98.

Ryder, H.E. and G.M. Heal, 1973. Optimal growth with intertemporally dependent preferences. The Review of Economic Studies, 40(1): 131.Available at: https://doi.org/10.2307/2296736.

Sargent, T.J., 1987. Some of Milton Friedman's scientific contributions to macroeconomics. Stanford, CA: Hoover Institution.

Seater, J.J., 1998. Testing the permanent-income/life-cycle hypothesis with aggregate data. Macroeconomic Dynamics, 2(3): 401-425.

Serlenga, L., 2001. Three alterative approaches to test the permanent income hypothesis indynamic panels. Department of Economics, University of Edinburgh. Available from https://pdfs.semanticscholar.org/91f9/643a3 137c7dad952954ca83eb02aafe275e3.pdf.

Takayama, A., 1985. Permanent income hypothesis: An optimal control theory approach to a classical macro thesis. Journal of Macroeconomics, 7(3): 347-362.Available at: https://doi.org/10.1016/0164-0704(85)90076-x.

Tekin, İ., 2011. Sensitivity of consumption to current income in developing countries: An empirical reinvestigation of absolute income hypothesis. Cukurova University Journal of the Institute of Social Sciences, 27(1): 48-62.

Turnovsky, S.J. and E. Burmeister, 1977. Perfect foresight, expectational consistency, and macroeconomic equilibrium. Journal of Political Economy, 85(2): 379-393.Available at: https://doi.org/10.1086/260568.

Uzawa, H., 1968. Time preferences, the consumption function, and optimum asset holdings. In J. N. Wolfe (Ed.), Value, Capital and Growth: Papers in Honour of Sir John Hicks. Edinburgh: University of Edinburgh Press. pp: 485-504.

Uzawa, H., 1990. Economic analysis. Tokyo: Iwanami (In Japanese).

Volterra, V., 1959. Theory of functionals and of integral and integro-differential equations. New York: Dover. 
Wan, H.Y., 1970. Optimal saving programs under intertemporally dependent preferences. International Economic Review, 11(3): 521 547.Available at: https://doi.org/10.2307/2525330.

Yaari, M.E., 1964. On the consumer's lifetime allocation process. International Economic Review, 5(3): 304-317.Available at: https://doi.org/10.2307/2525515. 